\title{
Hybrid control of Hopf bifurcation in a Lotka-Volterra predator-prey model with two delays
}

\author{
Miao Peng* ${ }^{*}$, Zhengdi Zhang and Xuedi Wang
}

\author{
"Correspondence: \\ pengmiao199101@126.com \\ Nonlinear Scientific Research \\ Center, Faculty of Science, Jiangsu \\ University, Zhenjiang, Jiangsu \\ 212013, P.R. China
}

\begin{abstract}
In this paper, the Hopf bifurcation control for a Lotka-Volterra predator-prey model with two delays is studied by using a hybrid control strategy. By analyzing the associated characteristic equation, its local stability and the existence of Hopf bifurcation with respect to both delays are established. In addition, the onset of an inherent bifurcation is delayed. Based on the normal form theory and the center manifold theorem, explicit formulas are derived to determine the direction of Hopf bifurcation and stability of the bifurcating periodic solution. Numerical simulation results confirm that the hybrid controller is efficient in controlling Hopf bifurcation.
\end{abstract}

Keywords: Hopf bifurcation; hybrid control; predator-prey model; stability

\section{Introduction}

In species dynamics, there are two kinds of mathematical models: the continuous temporal models described by differential equations and the discrete temporal models described by difference equations. The complex dynamics of these systems had attracted intensive as well as research attention in theoretical and mathematical biology during the last few decades. Some of the key discrete temporal models [1-5] and continuous temporal models [6-12] are referenced.

It is well known that significant theoretical development has recently been reported in the bifurcation theory of discrete temporal dynamic systems. There have been great and interesting predator-prey systems with time delay. This factor has induced more complicated dynamic characteristics than that without time delay because the presence of time delay causes a stable equilibrium to become unstable and subsequently the species to fluctuate. In [1], Han and Liu studied a discrete temporal model of Lotka-Volterra type with delay by a set of difference equations as follows:

$$
\left\{\begin{array}{l}
x_{k+1}=x_{k} \exp \left\{r_{1}-a_{11} x_{k-\tau}-a_{12} y_{k-\tau}\right\}, \\
y_{k+1}=y_{k} \exp \left\{r_{2}-a_{21} x_{k-\tau}-a_{22} y_{k-\tau}\right\},
\end{array} \quad \forall k \geq \tau,\right.
$$

where $x_{k}$ is the density of the first population at the $k$ th generation, $y_{k}$ denotes the density of the second population at the $k$ th generation, $r_{i}$ is the growth rate of population $i, a_{i j}$ $(i, j=1,2)$ stands for the intensity of intraspecific competition or interspecific action of

(c) The Author(s) 2017. This article is distributed under the terms of the Creative Commons Attribution 4.0 International License (http://creativecommons.org/licenses/by/4.0/), which permits unrestricted use, distribution, and reproduction in any medium, provided you give appropriate credit to the original author(s) and the source, provide a link to the Creative Commons license, and indicate if changes were made. 
species. They focus on the stability and bifurcation analysis and the direction analysis of the Neimark-Sacker bifurcations. This kind of discrete temporal model usually describes certain insects whose populations have non-overlapping generations or the number of populations is small in nature.

However, depending on the different species, some recent works showed that the continuous temporal models are more appropriate than the discrete temporal models when the populations have overlapping generations or the number of populations is big. In real situations, there are different time delays of species that affect the predator-prey systems. For instance, the species feedback time delay, the hunting delay, the gestation period of prey or predator, etc.

In this paper, we consider the following system with two delays described by differential equations with reference to $\mathrm{Xu}$ et al. [13]:

$$
\left\{\begin{array}{l}
\dot{x}(t)=x(t)\left[r_{1}-a_{11} x\left(t-\tau_{1}\right)-a_{12} y\left(t-\tau_{2}\right)\right], \\
\dot{y}(t)=y(t)\left[-r_{2}+a_{21} x\left(t-\tau_{2}\right)-a_{22} y\left(t-\tau_{1}\right)\right],
\end{array}\right.
$$

where $x(t)$ and $y(t)$ can be interpreted as the population densities of prey and predator at time $t, r_{1}>0$ represents an intrinsic growth rate of the prey and $r_{2}>0$ denotes the death rate of the predators; the parameters $a_{i j}(i, j=1,2)$ are all positive constants in which $a_{11}$ and $a_{22}$ represent the intraspecific competition rate of prey and predator, $a_{12}$ is the capturing rate, $a_{21} / a_{12}$ is the conversion rate of the predator, $\tau_{1}$ is the time delay due to the gestation of prey and predator, $\tau_{2}$ in the first equation of system (1.2) denotes the hunting delay of predator to prey and $\tau_{2}$ in the second equation of system (1.2) is the feedback delay of the predator to the growth of the species itself.

The dynamical behavior of the predator-prey systems with time delay has been studied comprehensively [14-22]. In the real world, there is sometimes a need to control a population at a reasonable level because otherwise this population may cause decrease or even extinction of other populations. With respect to the control of a biological system, the focus at present is on the state feedback control $[23,24]$ by changing the structure of the biocenose and by increasing the feeding pressure of the prey. For example, in order to eliminate algal bloom, an effective way is to introduce suitable fish species (chub etc.) that usually feed on plankton such that algal bloom can be controlled.

Hybrid control methods have been widely used by researchers [25-35]. Liu and Chung [27] proposed a hybrid control strategy for bifurcation in a continuous nonlinear dynamics system without time delay. Cheng and Cao [28] considered Hopf bifurcation control for a complex network model with time delays, and they used a hybrid control strategy to control the model. Chen et al. [30] proposed a new hybrid control strategy for microgrids with master-slave structure. In grid-connected operational mode, the droop control strategy was adopted for the main converter, while in stand-alone operational mode the droop gain would decrease to zero,thus becoming a conventional control. Ayadathil and Venkatesh [32] presented a hybrid control strategy for a matrix converter fed wind energy conversion system. Alfi et al. [35] investigated a hybrid control strategy for synchronization of a class of nonlinear chaotic systems by incorporating sliding mode control and state feedback control techniques via fuzzy logic. However, the hybrid control of bifurcation for a predator-prey system has not been extensively investigated. In this paper, a new control strategy of a system described in Eq. (1.2) is established. In the past, we mainly considered 
the state feedback control. For example, in order to eliminate the algal bloom, an effective way is to introduce a state feedback variable in the equation (such as chub) to change the system equilibrium. In fact, intraspecific effect coefficients, the interaction coefficients and others (e.g. temperature, irradiance, etc.) are affected by many factors. Furthermore, the parameters can be changed to regulate the system.

Motivated by Xu et al. [13] and based on a hybrid control by combining the state feedback control and perturbation parameter, the designing of a controller is established in this work in an effort to delay the occurrence of Hopf bifurcations in system (1.2). Here, a controlled system as follows is considered:

$$
\left\{\begin{array}{l}
\dot{x}(t)=\alpha x(t)\left[r_{1}-a_{11} x\left(t-\tau_{1}\right)-a_{12} y\left(t-\tau_{2}\right)\right]+\beta x\left(t-\tau_{1}\right), \\
\dot{y}(t)=\alpha y(t)\left[-r_{2}+a_{21} x\left(t-\tau_{2}\right)-a_{22} y\left(t-\tau_{1}\right)\right]+\beta y\left(t-\tau_{1}\right),
\end{array}\right.
$$

where $\alpha>0, \beta \in R$ is a control parameter. The parameters $x(t), y(t), a_{11}, a_{12}, a_{21}, a_{22}, r_{1}$, $r_{2}, \tau_{1}$ and $\tau_{2}$ are defined in system (1.2). $\beta x\left(t-\tau_{1}\right)$ and $\beta y\left(t-\tau_{1}\right)$ can affect the densities of prey and predator at time $t-\tau_{1}$, respectively, $\beta>0$ denotes increase in the quantity, while $\beta<0$ otherwise.

The biological meaning of system (1.3) can be interpreted as follows. In the absence of predators, the prey species follows the logistic equation $\dot{x}(t)=\alpha x(t)\left[r_{1}-a_{11}\left(x-\tau_{1}\right)\right]$, while in the presence of predators, there is a hunting delay $a_{12} y\left(t-\tau_{2}\right)$, with a certain delay $\tau_{2}$ called the hunting delay. In the absence of prey species, the predator species follows the equation $\dot{y}(t)=\alpha y(t)\left[-r_{2}-a_{22} y\left(t-\tau_{1}\right)\right]+\beta y\left(t-\tau_{1}\right)$. The positive feedback $a_{21} x\left(t-\tau_{2}\right)$ has a positive delay $\tau_{2}$ which is the delay in the predator maturation.

The remainder of this paper is organized as follows. In Section 2, the local stability and the existence of Hopf bifurcation at a positive equilibrium are discussed and the onset of an inherent bifurcation is delayed by analyzing the corresponding characteristic equations. The direction of Hopf bifurcation and the stability of bifurcating periodic solutions are derived in Section 3. In Section 4, numerical simulations are carried out to illustrate the validity of the main results. A brief conclusion is given in the last section.

\section{Local stability and Hopf bifurcation of the controlled system}

In this section, we shall investigate the stability of system (1.3) at the positive equilibrium and the existence of Hopf bifurcation by analyzing the corresponding linearized system.

System (1.3) has a unique positive equilibrium $E^{*}\left(x^{*}, y^{*}\right)$, where

$$
x^{*}=\frac{\left(\alpha r_{1}+\beta\right) a_{22}+\left(\alpha r_{2}-\beta\right) a_{12}}{\alpha\left(a_{11} a_{22}+a_{12} a_{21}\right)}, \quad y^{*}=\frac{\left(\alpha r_{1}+\beta\right) a_{21}-\left(\alpha r_{2}-\beta\right) a_{11}}{\alpha\left(a_{11} a_{22}+a_{12} a_{21}\right)},
$$

if the following condition

(H1) $\left(\alpha r_{1}+\beta\right) a_{22}-\left(\alpha r_{2}-\beta\right) a_{11}>0$

holds.

Let $\bar{x}(t)=x(t)-x^{*}, \bar{y}(t)=y(t)-y^{*}$ and denote $\bar{x}(t), \bar{y}(t)$ by $x(t), y(t)$, respectively, then system (1.3) becomes

$$
\left\{\begin{array}{l}
\dot{x}(t)=m_{1} x(t)+m_{2} x\left(t-\tau_{1}\right)+m_{3} y\left(t-\tau_{2}\right)+m_{4} x(t) x\left(t-\tau_{1}\right)+m_{5} x(t) y\left(t-\tau_{2}\right), \\
\dot{y}(t)=n_{1} y(t)+n_{2} x\left(t-\tau_{2}\right)+n_{3} y\left(t-\tau_{1}\right)+n_{4} x\left(t-\tau_{2}\right) y(t)+n_{5} y(t) y\left(t-\tau_{1}\right)
\end{array}\right.
$$


where

$$
\begin{aligned}
& m_{1}=\alpha\left(r_{1}-a_{11} x^{*}-a_{12} y^{*}\right), \quad m_{2}=-\alpha a_{11} x^{*}+\beta, \quad m_{3}=-\alpha a_{12} x^{*}, \\
& m_{4}=-\alpha a_{11}, \quad m_{5}=-\alpha a_{12}, \quad n_{1}=\alpha\left(-r_{2}+a_{21} x^{*}-a_{22} y^{*}\right), \\
& n_{2}=\alpha a_{21} y^{*}, \quad n_{3}=-\alpha a_{22} y^{*}+\beta, \quad n_{4}=\alpha a_{21}, \quad n_{5}=-\alpha a_{22} .
\end{aligned}
$$

Then we obtain a linearized system of system (2.1) as follows:

$$
\left\{\begin{array}{l}
\dot{x}(t)=m_{1} x(t)+m_{2} x\left(t-\tau_{1}\right)+m_{3} y\left(t-\tau_{2}\right), \\
\dot{y}(t)=n_{1} y(t)+n_{2} x\left(t-\tau_{2}\right)+n_{3} y\left(t-\tau_{1}\right) .
\end{array}\right.
$$

The corresponding characteristic equation of system (2.2) is

$$
\begin{aligned}
\lambda^{2}- & \left(m_{1}+n_{1}\right) \lambda+m_{1} n_{1}-\left(m_{2} \lambda+n_{3} \lambda-m_{1} n_{3}-m_{2} n_{1}\right) e^{-\lambda \tau_{1}} \\
& +m_{2} n_{3} e^{-2 \lambda \tau_{1}}-m_{3} n_{2} e^{-2 \lambda \tau_{2}}=0 .
\end{aligned}
$$

To investigate the root distribution of the transcendental equation (2.3), the result of Ruan and Wei [36] is introduced here.

Lemma 2.1 For the transcendental equation

$$
\begin{aligned}
p\left(\lambda, e^{-\lambda \tau_{1}}, \ldots, e^{-\lambda \tau_{m}}\right)= & \lambda^{n}+p_{1}^{(0)} \lambda^{n-1}+\cdots+p_{n-1}^{(0)} \lambda+p_{n}^{(0)} \\
& +\left[p_{1}^{(1)} \lambda^{n-1}+\cdots+p_{n-1}^{(1)} \lambda+p_{n}^{(1)}\right] e^{-\lambda \tau_{1}}+\cdots \\
& +\left[p_{1}^{(m)} \lambda^{n-1}+\cdots+p_{n-1}^{(m)} \lambda+p_{n}^{(m)}\right] e^{-\lambda \tau_{m}} \\
= & 0
\end{aligned}
$$

as $\left(\tau_{1}, \tau_{2}, \tau_{3}, \ldots, \tau_{m}\right)$ vary, the sum of orders of the zeros of $p\left(\lambda, e^{-\lambda \tau_{1}}, \ldots, e^{-\lambda \tau_{m}}\right)$ in the open right half plane can change, and only a zero appears on or crosses the imaginary axis.

In the sequel, we consider the following three cases.

Case (a) $\tau_{1}=\tau_{2}=0$, the characteristic equation (2.3) becomes

$$
\lambda^{2}-\left(m_{1}+m_{2}+n_{1}+n_{3}\right) \lambda+m_{1} n_{1}+m_{1} n_{3}+m_{2} n_{1}+m_{2} n_{3}-m_{3} n_{2}=0 .
$$

According to the Routh-Hurwitz criteria, a set of necessary and sufficient conditions for all roots of Eq. (2.4) to have a negative real part are given in the following form:

(H2) $m_{1}+m_{2}+n_{1}+n_{3}<0$.

Then the equilibrium point $E^{*}\left(x^{*}, y^{*}\right)$ is locally asymptotically stable when condition (H2) is satisfied.

Case (b) $\tau_{1}=0, \tau_{2}>0$, Eq. (2.3) reduces to

$$
\lambda^{2}+p \lambda+r+q e^{-2 \lambda \tau_{2}}=0
$$

where

$$
p=-\left(m_{1}+m_{2}+n_{1}+n_{3}\right), \quad q=-m_{3} n_{2}, \quad r=m_{1} n_{1}+m_{1} n_{3}+m_{2} n_{1}+m_{2} n_{3} .
$$


For $\omega>0$, suppose $i \omega$ is a root of Eq. (2.5), it follows that

$$
\left\{\begin{array}{l}
q \cos 2 \omega \tau_{2}=\omega^{2}-r \\
q \sin 2 \omega \tau_{2}=p \omega
\end{array}\right.
$$

which leads to

$$
\omega^{4}+\left(p^{2}-2 r\right) \omega^{2}+r^{2}-q^{2}=0
$$

It is easy to see that if the condition

(H3) $\left(p^{2}-2 r\right)>0, r^{2}-q^{2}>0$,

holds, then Eq. (2.7) has no positive roots. Hence, all roots of Eq. (2.5) have negative real parts when $\tau_{2} \in[0, \infty)$ under conditions $(\mathrm{H} 2)$ and $(\mathrm{H} 3)$. Further, if $(\mathrm{H} 2)$ and

(H4) $\left(p^{2}-2 r\right)>0, r^{2}-q^{2}<0$,

hold, then Eq. (2.7) has a unique positive root $\omega_{0}^{2}$. Substituting $\omega_{0}^{2}$ into Eq. (2.6), we obtain

$$
\tau_{2 n}=\frac{1}{2 \omega_{0}}\left\{\operatorname{arcos} \frac{\omega^{2}-r}{q}+2 n \pi\right\}, \quad n=0,1,2 \ldots
$$

According to the Hopf bifurcation theorem [37], we need to verify the transversality condition. Differentiating Eq. (2.5) with respect to $\tau_{2}$ and noticing that $\lambda$ is a function of $\tau_{2}$, we obtain

$$
\left(\frac{d \lambda}{d \tau_{2}}\right)^{-1}=\frac{(2 \lambda+p) e^{2 \lambda \tau_{2}}}{2 q \lambda}-\frac{\tau_{2}}{\lambda}
$$

which leads to

$$
\begin{aligned}
{\left[\frac{d(\operatorname{Re} \lambda)}{d \tau_{2}}\right]_{\tau_{2}=\tau_{2 n}} } & =\left.\operatorname{Re}\left\{\frac{(2 \lambda+p) e^{2 \lambda \tau_{2}}}{2 q \lambda}\right\}\right|_{\tau_{2}=\tau_{2 n}} \\
& =\frac{p \sin 2 \omega_{0} \tau_{2 n}+2 \omega_{0} \cos 2 \omega_{0} \tau_{2 n}}{q \omega_{0}} \\
& =\frac{p^{2}-2 r+2 \omega_{0}^{2}}{2 q^{2}}>0 .
\end{aligned}
$$

Noting that

$$
\left.\operatorname{sign}\left\{\frac{d(\operatorname{Re} \lambda)}{d \tau_{2}}\right\}\right|_{\tau_{2}=\tau_{2 n}}=\left.\operatorname{sign}\left\{\operatorname{Re}\left(\frac{d \lambda}{d \tau_{2}}\right)^{-1}\right\}\right|_{\tau_{2}=\tau_{2 n}}=1
$$

Hence, we have

$$
\left.\frac{d(\operatorname{Re} \lambda)}{d \tau_{2}}\right|_{\tau_{2}=\tau_{2 n}}>0
$$

According to the analysis above and Corollary 2.4 of [36], we have the following results.

Lemma 2.2 For $\tau_{1}=0$, assume that $(\mathrm{H} 1)$ and $(\mathrm{H} 2)$ are satisfied, then the following conclusions hold: 
(i) If (H3) holds, then the positive equilibrium $E^{*}\left(x^{*}, y^{*}\right)$ of system (1.3) is asymptotically stable for all $\tau_{2} \geq 0$.

(ii) If ( $\mathrm{H} 4)$ holds, then the positive equilibrium $E^{*}\left(x^{*}, y^{*}\right)$ of system (1.3) is asymptotically stable for all $\tau_{2} \in\left[0, \tau_{20}\right)$ and unstable for $\tau_{2}>\tau_{20}$. Furthermore, system (1.3)

undergoes a Hopf bifurcation at the positive equilibrium $E^{*}\left(x^{*}, y^{*}\right)$ when $\tau_{2}=\tau_{20}$.

Case (c) $\tau_{1}>0, \tau_{2}>0$. We consider (2.3) with $\tau_{2}$ in its stable interval, and $\tau_{1}$ is regarded as a parameter. Without loss of generality, we consider system (1.3) under assumptions $(\mathrm{H} 2)$ and $(\mathrm{H} 4)$. Let $i \omega(\omega>0)$ be the root of Eq. (2.3), then we obtain

$$
\begin{aligned}
& (i \omega)^{2}-\left(m_{1}+n_{1}\right) i \omega+m_{1} n_{1}-\left(m_{2} i \omega+n_{3} i \omega-m_{1} n_{3}-m_{2} n_{1}\right) e^{-i \omega \tau_{1}} \\
& +m_{2} n_{3} e^{-2 i \omega \tau_{1}}-m_{3} n_{2} e^{-2 i \omega \tau_{2}}=0
\end{aligned}
$$

Separating the real and imaginary parts, we have

$$
\left\{\begin{array}{l}
-k_{1} \sin \omega \tau_{1}+k_{2} \cos \omega \tau_{1}+k_{3} \cos 2 \omega \tau_{1}=\omega^{2}-m_{1} n_{1}+k_{4} \cos 2 \omega \tau_{2} \\
-k_{1} \cos \omega \tau_{1}-k_{2} \sin \omega \tau_{1}-k_{3} \sin 2 \omega \tau_{1}=\left(m_{1}+n_{1}\right) \omega-k_{4} \sin 2 \omega \tau_{2}
\end{array}\right.
$$

where

$$
\begin{aligned}
& k_{1}=\omega\left(m_{2}+n_{3}\right), \quad k_{2}=m_{1} n_{3}+m_{2} n_{1}, \\
& k_{3}=m_{2} n_{3}, \quad k_{4}=m_{3} n_{2},
\end{aligned}
$$

which lead to

$$
2 k_{1} k_{3} \sin \omega \tau_{1}+2 k_{2} k_{3} \cos \omega \tau_{1}=k
$$

where

$$
\begin{aligned}
k= & \omega^{4}+\left(m_{1}^{2}+n_{1}^{2}+2 k_{4} \cos 2 \omega \tau_{2}\right) \omega^{2}-2 k_{4}\left(m_{1}+n_{1}\right) \sin 2 \omega \tau_{2} \omega \\
& -2 m_{1} n_{1} k_{4} \cos 2 \omega \tau_{2}+m_{1}^{2} n_{1}^{2}+k_{4}^{2}-k_{1}^{2}-k_{2}^{2}-k_{3}^{2} .
\end{aligned}
$$

Since $\sin \omega \tau_{1}= \pm \sqrt{1-\cos ^{2} \omega \tau_{1}}$, we consider the following two cases:

(i) $\sin \omega \tau_{1}=\sqrt{1-\cos ^{2} \omega \tau_{1}}$, Eq. (2.11) becomes

$$
2 k_{1} k_{3} \sqrt{1-\cos ^{2} \omega \tau_{1}}+2 k_{2} k_{3} \cos \omega \tau_{1}=k
$$

It is easy to compute $\cos \omega \tau_{1}$ by Eq. (2.12) noting that

$$
\cos \omega \tau_{1}=f_{1}(\omega), \quad \sin \omega \tau_{1}=f_{2}(\omega), \quad f_{1}^{2}(\omega)+f_{2}^{2}(\omega)=1
$$

Hence, we can determine

$$
\tau_{1_{1}}^{(n)}=\frac{1}{\omega}\left[\arccos f_{1}(w)+2 n \pi\right] \quad(n=0,1,2, \ldots)
$$

and $\omega$ is a root of $f_{1}^{2}(\omega)+f_{2}^{2}(\omega)=1$. 
(ii) $\sin \omega \tau_{1}=-\sqrt{1-\cos ^{2} \omega \tau_{1}}$, Eq. (2.11) becomes

$$
-2 k_{1} k_{3} \sqrt{1-\cos ^{2} \omega \tau_{1}}+2 k_{2} k_{3} \cos \omega \tau_{1}=k
$$

It is easy to compute $\cos \omega \tau_{1}$ by Eq. (2.13), noting that

$$
\cos \omega \tau_{1}=f_{1}^{*}(\omega), \quad \sin \omega \tau_{1}=f_{2}^{*}(\omega), \quad f_{1}^{* 2}(\omega)+f_{2}^{* 2}(\omega)=1
$$

Hence, we can determine

$$
\tau_{1_{2}}^{(n)}=\frac{1}{\omega}\left[\arccos f_{1}^{*}(w)+2 n \pi\right] \quad(n=0,1,2, \ldots),
$$

and $\omega$ is a root of $f_{1}^{* 2}(\omega)+f_{2}^{* 2}(\omega)=1$.

Let

$$
\tau_{1_{0}}=\min \left\{\tau_{1_{1}}^{(n)}, \tau_{1_{2}}^{(n)}\right\} \quad(n=0,1,2, \ldots)
$$

hence, for $\tau_{2} \in\left[0, \tau_{2_{0}}\right)$, Eq. (2.3) has a pair of purely imaginary roots $\pm i \omega^{*}$ when $\tau_{1}=\tau_{1_{0}}$.

In the following, we assume that

(H5) $\left[\frac{d(\operatorname{Re} \lambda)}{d \tau_{1}}\right]_{\lambda=i \omega^{*}} \neq 0$.

We have the following theorem.

Theorem 2.1 If conditions (H1), (H2), (H4) and (H5) hold and $\tau_{2} \in\left[0, \tau_{2_{0}}\right)$, then the positive equilibrium $E^{*}\left(x^{*}, y^{*}\right)$ of system $(1.3)$ is asymptotically stable for $\tau_{1} \in\left[0, \tau_{1_{0}}\right)$ and unstable when $\tau_{1}>\tau_{1_{0}}$. Furthermore, system (1.3) undergoes Hopf bifurcation at $\tau_{1}=\tau_{1_{0}}$.

\section{Direction and stability of Hopf bifurcation of the controlled system}

In the previous section, we have shown that the controlled system (1.3) undergoes Hopf bifurcation for different combinations of $\tau_{1}$ and $\tau_{2}$. In this section, we will investigate the direction of Hopf bifurcation and the stability of bifurcating periodic solutions of the controlled system (1.3). Throughout this section, we assume that system (1.3) undergoes a Hopf bifurcation for $\tau_{2}^{*} \in\left(0, \tau_{2_{0}}\right)$ and $\tau_{1}=\tau_{1_{0}}$. The theoretical approach we apply is based on the normal form theory and center manifold theory [37].

Without loss of generality, we assume that $\tau_{2}^{*}<\tau_{1_{0}}$, where $\tau_{2}^{*} \in\left(0, \tau_{2_{0}}\right)$. For convenience, let $\bar{u}_{i}(t)=u_{i}(\tau t)(i=1,2)$ and $\tau_{1}=\tau_{1_{0}}+\mu$, where $\tau_{1_{0}}$ is defined by Eq. (2.14) and $\mu \in R$, then system (1.3) can be written as a functional differential equation (FDE) in $C=C\left([-1,0], R^{2}\right)$ :

$$
u^{\prime}(t)=L_{\mu}\left(u_{t}\right)+F\left(\mu, u_{t}\right)
$$

where $u(t)=(x(t), y(t))^{T} \in C, u_{t}(\theta)=u(t+\theta)=(x(t+\theta), y(t+\theta))^{T} \in C$, and $L_{\mu}: C \rightarrow R$, $F: R \times C \rightarrow R$ are given by

$$
L_{\mu}(\varphi)=\left(\tau_{1}+\mu\right) B\left(\begin{array}{l}
\varphi_{1}(0) \\
\varphi_{2}(0)
\end{array}\right)+\left(\tau_{1}+\mu\right) C\left(\begin{array}{c}
\varphi_{1}\left(-\frac{\tau_{2}^{*}}{\tau_{1}}\right) \\
\varphi_{2}\left(-\frac{\tau_{2}^{*}}{\tau_{1_{0}}}\right)
\end{array}\right)+\left(\tau_{1}+\mu\right) D\left(\begin{array}{c}
\varphi_{1}(-1) \\
\varphi_{2}(-1)
\end{array}\right)
$$


and

$$
F(\mu, \varphi)=\left(\tau_{1}+\mu\right)\left(f_{1}, f_{2}\right)^{T},
$$

with

$$
B=\left(\begin{array}{cc}
m_{1} & 0 \\
0 & n_{1}
\end{array}\right), \quad C=\left(\begin{array}{cc}
0 & m_{3} \\
n_{2} & 0
\end{array}\right), \quad D=\left(\begin{array}{cc}
m_{2} & 0 \\
0 & n_{3}
\end{array}\right),
$$

and

$$
\begin{aligned}
& f_{1}=m_{4} \varphi_{1}(0) \varphi_{1}(-1)+m_{5} \varphi_{1}(0) \varphi_{2}\left(-\frac{\tau_{2}^{*}}{\tau_{1_{0}}}\right), \\
& f_{2}=n_{4} \varphi_{1}\left(-\frac{\tau_{2}^{*}}{\tau_{1_{0}}}\right) \varphi_{2}(0)+n_{5} \varphi_{2}(0) \varphi_{2}(-1) .
\end{aligned}
$$

By the Riesz representation theorem, there exists a $2 \times 2$ matrix function $\eta(\theta, \mu), \theta \in$ $[-1,0]$, whose elements are of bounded variation, such that

$$
L_{\mu} \varphi=\int_{-1}^{0} d \eta(\theta, \mu) \varphi(\theta) \quad \text { for } \varphi \in C
$$

In fact, we can choose

$$
\eta(\theta, \mu)= \begin{cases}\left(\tau_{1_{0}}+\mu\right)(B+C+D), & \theta=0, \\ \left(\tau_{1_{0}}+\mu\right)(C+D), & \theta \in\left[-\frac{\tau_{2}^{*}}{\tau_{1}}, 0\right), \\ \left(\tau_{1_{0}}+\mu\right) D, & \theta \in\left(-1,-\frac{\tau_{2}^{*}}{\tau_{1_{0}}}\right), \\ 0, & \theta=-1 .\end{cases}
$$

For $\varphi \in C\left([-1,0], R^{2}\right)$, define

$$
A(\mu) \varphi= \begin{cases}\frac{d \varphi(\theta)}{d \theta}, & -1 \leq \theta<0, \\ \int_{-1}^{0} d \eta(s, \mu) \varphi(s), & \theta=0\end{cases}
$$

and

$$
R_{\mu}(\varphi)= \begin{cases}0, & -1 \leq \theta<0 \\ F(\mu, \varphi), & \theta=0\end{cases}
$$

Then Eq. (3.1) can be transformed into the following operator equation:

$$
u_{t}^{\prime}=A(\mu) u_{t}+R(\mu) u_{t}
$$

where $u_{t}=u(t+\theta)=\left(u_{1}(t+\theta), u_{2}(t+\theta)\right), \theta \in[-1,0]$.

For $\phi \in C\left([-1,0],\left(R^{2}\right)^{*}\right)$, where $\left(R^{2}\right)^{*}$ is the 2-dimensional space of row vectors, we further define the adjoint operator $A^{*}$ of $A(0)$ :

$$
A^{*} \phi(s)= \begin{cases}-\frac{d \phi(s)}{d s}, & s \in(0,1] \\ \int_{-1}^{0} d \eta^{T}(t, 0) \phi(-t), & s=0\end{cases}
$$


For $\varphi \in C\left([-1,0], R^{2}\right)$ and $\phi \in C\left([-1,0],\left(R^{2}\right)^{*}\right)$, define the bilinear form

$$
\langle\phi(s), \varphi(s)\rangle=\bar{\phi}(0) \varphi(0)-\int_{-1}^{0} \int_{\xi=0}^{\theta} \phi(\xi-\theta) d \eta(\theta) \varphi(\xi) d \xi,
$$

where $\eta(\theta)=\eta(\theta, 0), A=A(0)$ and $A^{*}$ are adjoint operators. From Section 2, we know that $\pm i \omega^{*} \tau_{1_{0}}$ are eigenvalues of $A(0)$, and they are also the eigenvalues of $A^{*}$ corresponding to $i \omega^{*} \tau_{1_{0}}$ and $-i \omega^{*} \tau_{1_{0}}$. Further, we suppose $q(\theta)=(1, \alpha)^{T} e^{i \omega^{*} \tau_{1} \theta}$ is the eigenvector of $A(0)$ corresponding to $i \omega^{*} \tau_{1_{0}}$ and $q^{*}(s)=M\left(1, \alpha^{*}\right) e^{i \omega^{*} \tau_{1} s}$ is the eigenvector of $A^{*}$ corresponding to $-i \omega^{*} \tau_{1_{0}}$, where $M=1 / D$.

By the direct calculation, we obtain

$$
\begin{aligned}
& \alpha=\frac{i \omega^{*}-m_{1}-m_{2} e^{-i \omega^{*} \tau_{1}}}{m_{3} e^{-i \omega^{*} \tau_{2}^{*}}}, \quad \alpha^{*}=-\frac{i \omega^{*}+m_{1}+m_{2} e^{-i \omega^{*} \tau_{1}}}{n_{2} e^{-i \omega^{*} \tau_{2}^{*}}}, \\
& D=1+\bar{\alpha} \alpha^{*}+m_{2} \tau_{1_{0}} e^{i \omega^{*} \tau_{1}}+n_{2} \alpha^{*} \tau_{2}^{*} e^{i \omega^{*} \tau_{2}^{*}}+m_{3} \bar{\alpha} \tau_{2}^{*} e^{i \omega^{*} \tau_{2}^{*}}+n_{3} \bar{\alpha} \alpha^{*} \tau_{1_{0}} e^{i \omega^{*} \tau_{1}} .
\end{aligned}
$$

Then we have $\left\langle q^{*}(s), q(\theta)\right\rangle=1,\left\langle q^{*}(s), \bar{q}(\theta)\right\rangle=0$.

Next, we use the same notations as those in Hassard [37] and firstly compute the coordinates to describe the center manifold $C_{0}$ at $\mu=0$. Let $u_{t}$ be the solution of Eq. (3.1) when $\mu=0$.

Define

$$
\begin{aligned}
& z(t)=\left\langle q^{*}, u_{t}\right\rangle, \\
& W(t, \theta)=u_{t}(\theta)-2 \operatorname{Re}\{z(t) q(\theta)\},
\end{aligned}
$$

on the center manifold $C_{0}$, and we have

$$
W(t, \theta)=W(z(t), \bar{z}(t), \theta),
$$

where

$$
W(z(t), \bar{z}(t), \theta)=W(z, \bar{z})=W_{20} \frac{z^{2}}{2}+W_{11} z \bar{z}+W_{02} \frac{\bar{z}^{2}}{2}+\cdots
$$

and $z$ and $\bar{z}$ are the local coordinates for the center manifold $C_{0}$ in the direction of $q^{*}$ and $\bar{q}^{*}$. Noting that $W$ is also real if $u_{t}$ is real, we consider real solutions. For solutions $u_{t} \in C_{0}$ of Eq. (3.1),

$$
\dot{z}(t)=i \omega^{*} \tau_{1_{0}} z+\bar{q}^{*}(\theta) F(0, W(z, \bar{z}, \theta))+2 \operatorname{Re}\{z q(\theta)\} .
$$

We define this equation as

$$
\dot{z}(t)=i \omega^{*} \tau_{1_{0}} z+\bar{q}^{*}(0) F_{0}
$$

That is,

$$
\dot{z}(t)=i \omega^{*} \tau_{10} z+g(z, \bar{z})
$$


where

$$
\begin{aligned}
g(z, \bar{z}) & =\bar{q}^{*}(0) F_{0}(z, \bar{z})=F\left(0, u_{t}\right) \\
& =g_{20} \frac{z^{2}}{2}+g_{11} z \bar{z}+g_{02} \frac{\bar{z}^{2}}{2}+g_{21} \frac{z^{2} \bar{z}}{2}+\cdots
\end{aligned}
$$

Noticing $u_{t}(\theta)=\left(x_{t}(\theta), y_{t}(\theta)\right)=W(t, \theta)+z q(\theta)+\bar{z} \bar{q}(\theta)$ and $q(\theta)=(1, \alpha)^{T} e^{i \omega^{*} \tau_{1} \theta}$ by Eq. (3.9), we have

$$
\begin{aligned}
& x_{t}(0)=z+\bar{z}+\frac{1}{2} W_{20}^{(1)}(0) z^{2}+W_{11}^{(1)}(0) z \bar{z}+\frac{1}{2} W_{02}^{(1)}(0) \bar{z}^{2}+\cdots, \\
& y_{t}(0)=\alpha z+\bar{\alpha} \bar{z}+\frac{1}{2} W_{20}^{(2)}(0) z^{2}+W_{11}^{(2)}(0) z \bar{z}+\frac{1}{2} W_{02}^{(2)}(0) \bar{z}^{2}+\cdots, \\
& x_{t}(-1)=z e^{-i \omega^{*} \tau_{1}}+\bar{z} e^{i \omega^{*} \tau_{1}}+\frac{1}{2} W_{20}^{(1)}(-1) z^{2}+W_{11}^{(1)}(-1) z \bar{z}+\frac{1}{2} W_{02}^{(1)}(-1) \bar{z}^{2}+\cdots, \\
& y_{t}(-1)=\alpha z e^{-i \omega^{*} \tau_{1}}+\bar{\alpha} \bar{z} e^{i \omega^{*} \tau_{1}}+\frac{1}{2} W_{20}^{(2)}(-1) z^{2}+W_{11}^{(2)}(-1) z \bar{z}+\frac{1}{2} W_{02}^{(2)}(-1) \bar{z}^{2}+\cdots, \\
& x_{t}\left(-\frac{\tau_{2}^{*}}{\tau_{1_{0}}}\right)=z e^{-i \omega^{*} \tau_{2}^{*}}+\bar{z} e^{i \omega^{*} \tau_{2}^{*}}+\frac{1}{2} W_{20}^{(1)}\left(-\frac{\tau_{2}^{*}}{\tau_{1_{0}}}\right) z^{2}+W_{11}^{(1)}\left(-\frac{\tau_{2}^{*}}{\tau_{1_{0}}}\right) z \bar{z} \\
& +\frac{1}{2} W_{02}^{(1)}\left(-\frac{\tau_{2}^{*}}{\tau_{1_{0}}}\right) \bar{z}^{2}+\cdots, \\
& y_{t}\left(-\frac{\tau_{2}^{*}}{\tau_{1_{0}}}\right)=\alpha z e^{-i \omega^{*} \tau_{2}^{*}}+\bar{\alpha} \bar{z} e^{i \omega^{*} \tau_{2}^{*}}+\frac{1}{2} W_{20}^{(2)}\left(-\frac{\tau_{2}^{*}}{\tau_{1_{0}}}\right) z^{2}+W_{11}^{(2)}\left(-\frac{\tau_{2}^{*}}{\tau_{1_{0}}}\right) z \bar{z} \\
& +\frac{1}{2} W_{02}^{(2)}\left(-\frac{\tau_{2}^{*}}{\tau_{1_{0}}}\right) \bar{z}^{2}+\cdots .
\end{aligned}
$$

Then from Eq. (3.12) we have

$$
\begin{aligned}
g(z, \bar{z})= & \bar{M} \tau_{1_{0}}\left[\left(m_{4} e^{-i \omega^{*} \tau_{1}}+m_{5} \alpha e^{-i \omega^{*} \tau_{2}^{*}}\right)+\bar{\alpha}^{*}\left(n_{4} \alpha e^{-i \omega^{*} \tau_{2}^{*}}+n_{5} \alpha^{2} e^{-i \omega^{*} \tau_{1_{0}}}\right)\right] z^{2} \\
& +2 \bar{M} \tau_{1_{0}}\left[\left(m_{4} \operatorname{Re}\left\{e^{-i \omega^{*} \tau_{1}}\right\}+m_{5} \operatorname{Re}\left\{\alpha e^{-i \omega^{*} \tau_{2}^{*}}\right\}\right)\right. \\
& \left.+\bar{\alpha}^{*}\left(n_{4} \operatorname{Re}\left\{\alpha e^{i \omega^{*} \tau_{2}^{*}}\right\}+n_{5} \operatorname{Re}\left\{|\alpha|^{2} e^{i \omega^{*} \tau_{1}}\right\}\right)\right] z \bar{z} \\
& +\bar{M} \tau_{1_{0}}\left[\left(m_{4} e^{i \omega^{*} \tau_{1}}+m_{5} \bar{\alpha} e^{i \omega^{*} \tau_{2}^{*}}\right)+\bar{\alpha}^{*}\left(n_{4} \bar{\alpha} e^{i \omega \tau_{2}^{*}}+n_{5} \bar{\alpha}^{2} e^{i \omega^{*} \tau_{1}}\right)\right] \bar{z}^{2} \\
& +\bar{M} \tau_{1_{0}}\left[m_{4}\left(W_{11}^{(1)}(-1)+\frac{1}{2} W_{20}^{(1)}(-1)+\frac{1}{2} W_{20}^{(1)}(0) e^{i \omega^{*} \tau_{1}}+W_{11}^{(1)}(0) e^{-i \omega^{*} \tau_{1}}\right)\right. \\
& +m_{5}\left(W_{11}^{(2)}\left(-\frac{\tau_{2}^{*}}{\tau_{1_{0}}}\right)+\frac{1}{2} W_{20}^{(2)}\left(-\frac{\tau_{2}^{*}}{\tau_{1_{0}}}\right)+\frac{1}{2} W_{20}^{(1)}(0) \bar{\alpha} e^{i \omega^{*} \tau_{2}^{*}}+W_{11}^{(1)}(0) \alpha e^{-i \omega^{*} \tau_{2}^{*}}\right) \\
& +\bar{\alpha}^{*}\left[n _ { 4 } \left(W_{11}^{(2)}(0) e^{-i \omega^{*} \tau_{2}^{*}}+\frac{1}{2} W_{20}^{(2)}(0) e^{i \omega^{*} \tau_{2}^{*}}\right.\right. \\
& \left.+\frac{1}{2} \bar{\alpha} W_{20}^{(1)}\left(-\frac{\tau_{2}^{*}}{\tau_{1_{0}}}\right)+\alpha W_{11}^{(1)}\left(-\frac{\tau_{2}^{*}}{\tau_{1_{0}}}\right)\right) \\
& +n_{5}\left(\alpha W_{11}^{(2)}(-1)+\frac{1}{2} \bar{\alpha} W_{20}^{(2)}(-1)\right. \\
& \left.\left.\left.+\frac{1}{2} \bar{\alpha} W_{20}^{(2)}(0) e^{i \omega^{*} \tau_{1}}+\alpha W_{11}^{(2)}(0) e^{-i \omega^{*} \tau_{1_{0}}}\right)\right]\right] z^{2} \bar{z}+\cdots
\end{aligned}
$$


Comparing the coefficients with Eq. (3.12), we obtain

$$
\begin{aligned}
& g_{20}=2 \bar{M} \tau_{1_{0}}\left[\left(m_{4} e^{-i \omega^{*} \tau_{1}}+m_{5} \alpha e^{-i \omega^{*} \tau_{2}^{*}}\right)+\bar{\alpha}^{*}\left(n_{4} \alpha e^{-i \omega^{*} \tau_{2}^{*}}+n_{5} \alpha^{2} e^{-i \omega^{*} \tau_{10}}\right)\right], \\
& g_{11}=2 \bar{M} \tau_{1_{0}}\left[\left(m_{4} \operatorname{Re}\left\{e^{-i \omega^{*} \tau_{1}}\right\}+m_{5} \operatorname{Re}\left\{\alpha e^{-i \omega^{*} \tau_{2}^{*}}\right\}\right)\right. \\
& \left.+\bar{\alpha}^{*}\left(n_{4} \operatorname{Re}\left\{\alpha e^{i \omega^{*} \tau_{2}^{*}}\right\}+n_{5} \operatorname{Re}\left\{|\alpha|^{2} e^{i \omega^{*} \tau_{1}}\right\}\right)\right], \\
& g_{02}=2 \bar{M} \tau_{1_{0}}\left[\left(m_{4} e^{i \omega^{*} \tau_{1}}+m_{5} \bar{\alpha} e^{i \omega^{*} \tau_{2}^{*}}\right)+\bar{\alpha}^{*}\left(n_{4} \bar{\alpha} e^{i \omega^{*} \tau_{2}^{*}}+n_{5} \bar{\alpha}^{2} e^{i \omega^{*} \tau_{1}}\right)\right], \\
& g_{21}=2 \bar{M} \tau_{1_{0}}\left[m_{4}\left(W_{11}^{(1)}(-1)+\frac{1}{2} W_{20}^{(1)}(-1)+\frac{1}{2} W_{20}^{(1)}(0) e^{i \omega^{*} \tau_{1}}+W_{11}^{(1)}(0) e^{-i \omega^{*} \tau_{1}}\right)\right. \\
& +m_{5}\left(W_{11}^{(2)}\left(-\frac{\tau_{2}^{*}}{\tau_{1_{0}}}\right)+\frac{1}{2} W_{20}^{(2)}\left(-\frac{\tau_{2}^{*}}{\tau_{1_{0}}}\right)+\frac{1}{2} W_{20}^{(1)}(0) \bar{\alpha} e^{i \omega^{*} \tau_{2}^{*}}+W_{11}^{(1)}(0) \alpha e^{-i \omega^{*} \tau_{2}^{*}}\right) \\
& +\bar{\alpha}^{*}\left[n_{4}\left(W_{11}^{(2)}(0) e^{-i \omega^{*} \tau_{2}^{*}}+\frac{1}{2} W_{20}^{(2)}(0) e^{i \omega^{*} \tau_{2}^{*}}+\frac{1}{2} \bar{\alpha} W_{20}^{(1)}\left(-\frac{\tau_{2}^{*}}{\tau_{1_{0}}}\right)+\alpha W_{11}^{(1)}\left(-\frac{\tau_{2}^{*}}{\tau_{1_{0}}}\right)\right)\right. \\
& \left.\left.+n_{5}\left(\alpha W_{11}^{(2)}(-1)+\frac{1}{2} \bar{\alpha} W_{20}^{(2)}(-1)+\frac{1}{2} \bar{\alpha} W_{20}^{(2)}(0) e^{i \omega^{*} \tau_{1}}+\alpha W_{11}^{(2)}(0) e^{-i \omega^{*} \tau_{1}}\right)\right]\right] \text {. }
\end{aligned}
$$

Since there are $W_{20}(\theta)$ and $W_{11}(\theta)$ in $g_{21}$, in the sequel, we shall compute these quantities. From Eqs. (3.8) and (3.9), we have

$$
\begin{aligned}
W^{\prime} & = \begin{cases}A W-2 \operatorname{Re}\left\{\bar{q}^{*}(0) F_{0} q(\theta)\right\}, & -1 \leq \theta<0, \\
A W-2 \operatorname{Re}\left\{\bar{q}^{*}(0) F_{0} q(\theta)\right\}+F_{0}, & \theta=0,\end{cases} \\
& =A W+H(z, \bar{z}, \theta),
\end{aligned}
$$

where

$$
H(z, \bar{z}, \theta)=H_{20}(\theta) \frac{z^{2}}{2}+H_{11}(\theta) z \bar{z}+H_{02}(\theta) \frac{\bar{z}^{2}}{2}+H_{21}(\theta) \frac{z^{2} \bar{z}}{2}+\cdots
$$

Comparing the coefficients, we obtain

$$
\begin{aligned}
& \left(A W-2 i \tau_{1_{0}} \omega^{*}\right) W_{20}=-H_{20}(\theta), \\
& A W_{11}(\theta)=-H_{11}(\theta) .
\end{aligned}
$$

From Eq. (3.13), we know that for $\theta \in[-1,0)$,

$$
H(z, \bar{z}, \theta)=-\bar{q}^{*}(0) f_{0} q(\theta)-q^{*}(0) \bar{f}_{0} \bar{q}(\theta)=-g q(\theta)-\bar{g} \bar{q}(\theta) .
$$

Comparing the coefficients with Eq. (3.14) gives

$$
H_{20}(\theta)=-g_{20} q(\theta)-\bar{g}_{02} \bar{q}(\theta)
$$

and

$$
H_{11}(\theta)=-g_{11} q(\theta)-\bar{g}_{11} \bar{q}(\theta)
$$


From Eqs. (3.15), (3.18) and the definition of $A$, it follows that

$$
W_{20}^{\prime}(\theta)=2 i \omega^{*} \tau_{1_{0}} W_{20}(\theta)+g_{20} q(\theta)+\bar{g}_{02} \bar{q}(\theta)
$$

Notice that $q(\theta)=(1, \alpha)^{T} e^{i \omega^{*} \tau_{1} \theta}$, hence

$$
W_{20}(\theta)=\frac{i g_{20}}{\omega^{*} \tau_{1_{0}}} q(0) e^{i \omega^{*} \tau_{10} \theta}+\frac{i \bar{g}_{02}}{3 \omega^{*} \tau_{1_{0}}} \bar{q}(0) e^{-i \omega^{*} \tau_{1} \theta}+E_{1} e^{2 i \omega^{*} \tau_{1} \theta},
$$

where $E_{1}=\left(E_{1}^{(1)}, E_{1}^{(2)}\right)^{T} \in R^{2}$ is a constant vector. Similarly, from Eqs. (3.16) and (3.19), we obtain

$$
\begin{aligned}
& W_{11}^{\prime}(\theta)=g_{11} q(\theta)+\bar{g}_{11} \bar{q}(\theta), \\
& W_{11}(\theta)=-\frac{i g_{11}}{\omega^{*} \tau_{1_{0}}} q(0) e^{i \omega^{*} \tau_{10} \theta}+\frac{i \bar{g}_{11}}{\omega^{*} \tau_{1_{0}}} \bar{q}(0) e^{-i \omega^{*} \tau_{10} \theta}+E_{2},
\end{aligned}
$$

where $E_{2}=\left(E_{2}^{(1)}, E_{2}^{(2)}\right)^{T} \in R^{2}$ is also a constant vector.

In what follows, we shall seek appropriate $E_{1}$ and $E_{2}$ in Eqs. (3.21) and (3.23), respectively. It follows from the definition of $A$ and Eqs. (3.18), (3.19) that

$$
\int_{-1}^{0} d \eta(\theta) W_{20}(\theta)=2 i \omega^{*} \tau_{1_{0}} W_{20}(0)-H_{20}(0)
$$

and

$$
\int_{-1}^{0} d \eta(\theta) W_{11}(\theta)=-H_{11}(0),
$$

where $\eta(\theta)=\eta(0, \theta)$. From Eqs. (3.15) and (3.16) we have

$$
\begin{aligned}
& H_{20}(0)=-g_{20}(0) q(0)-\bar{g}_{02}(0) \bar{q}(0)+2 \tau_{1_{0}}\left(H_{1}, H_{2}\right)^{T}, \\
& H_{11}(0)=-g_{11}(0) q(0)-\bar{g}_{11}(0) \bar{q}(0)+2 \tau_{1_{0}}\left(p_{1}, p_{2}\right)^{T},
\end{aligned}
$$

where

$$
\begin{aligned}
& H_{1}=m_{4} e^{-i \omega^{*} \tau_{1}}+m_{5} \alpha e^{-i \omega^{*} \tau_{2}^{*},} \\
& H_{2}=n_{4} \alpha e^{-i \omega^{*} \tau_{2}^{*}}+n_{5} \alpha^{2} e^{-i \omega^{*} \tau_{1}}, \\
& p_{1}=m_{4} \operatorname{Re}\left\{e^{-i \omega^{*} \tau_{1}}\right\}+m_{5} \operatorname{Re}\left\{\alpha e^{-i \omega^{*} \tau_{2}^{*}}\right\}, \\
& p_{2}=n_{4} \operatorname{Re}\left\{\alpha e^{i \omega^{*} \tau_{2}^{*}}\right\}+n_{5} \operatorname{Re}\left\{|\alpha|^{2} e^{i \omega^{*} \tau_{1}}\right\} .
\end{aligned}
$$

Noting that

$$
\begin{aligned}
& \left(i \omega^{*} \tau_{1_{0}} I-\int_{-1}^{0} e^{i \omega^{*} \tau_{1_{0}} \theta} d \eta(\theta)\right) q(0)=0, \\
& \left(-i \omega^{*} \tau_{1_{0}} I-\int_{-1}^{0} e^{-i \omega^{*} \tau_{0} \theta} d \eta(\theta)\right) \bar{q}(0)=0,
\end{aligned}
$$


and substituting Eqs. (3.21) and (3.26) into Eq. (3.24), we have

$$
\left(2 i \omega^{*} \tau_{1_{0}} I-\int_{-1}^{0} e^{2 i \omega^{*} \tau_{1_{0}} \theta} d \eta(\theta)\right) E_{1}=2 \tau_{1_{0}}\left(H_{1}, H_{2}\right)^{T} .
$$

That is,

$$
\left(\begin{array}{cc}
2 i \omega^{*}-m_{1}-m_{2} e^{-2 i \omega^{*} \tau_{1}} & -m_{3} e^{-2 i \omega^{*} \tau_{2}^{*}} \\
-n_{2} e^{-2 i \omega^{*} \tau_{2}^{*}} & 2 i \omega^{*}-n_{1}-n_{3} e^{-2 i \omega^{*} \tau_{1}}
\end{array}\right) E_{1}=2\left(H_{1}, H_{2}\right)^{T} .
$$

It follows that

$$
E_{1}^{(1)}=\frac{\Delta_{11}}{\Delta_{1}}, \quad E_{1}^{(2)}=\frac{\Delta_{12}}{\Delta_{1}},
$$

with

$$
\Delta_{1}=\operatorname{det}\left(\begin{array}{ll}
v_{1} & v_{2} \\
v_{3} & v_{4}
\end{array}\right), \quad \Delta_{11}=2 \operatorname{det}\left(\begin{array}{ll}
H_{1} & v_{2} \\
H_{2} & v_{4}
\end{array}\right), \quad \Delta_{12}=2 \operatorname{det}\left(\begin{array}{ll}
v_{1} & H_{1} \\
v_{3} & H_{2}
\end{array}\right)
$$

where

$$
\begin{aligned}
& v_{1}=2 i \omega^{*}-m_{1}-m_{2} e^{-2 i \omega^{*} \tau_{1}}, \quad \nu_{2}=-m_{3} e^{-2 i \omega^{*} \tau_{2}^{*},} \quad \nu_{3}=-n_{2} e^{-2 i \omega^{*} \tau_{2}^{*}}, \\
& v_{4}=2 i \omega^{*}-n_{1}-n_{3} e^{-2 i \omega^{*} \tau_{1}} .
\end{aligned}
$$

Similarly, substituting Eqs. (3.22) and (3.27) into Eq. (3.25), we have

$$
\left(\int_{-1}^{0} d \eta(\theta)\right) E_{2}=2 \tau_{1_{0}}\left(p_{1}, p_{2}\right)^{T}
$$

that is,

$$
\left(\begin{array}{cc}
m_{1}+m_{2} & m_{3} \\
n_{2} & n_{1}+n_{3}
\end{array}\right) E_{2}=2\left(-p_{1},-p_{2}\right)^{T}
$$

It follows that

$$
E_{2}^{(1)}=\frac{\Delta_{21}}{\Delta_{2}}, \quad E_{2}^{(2)}=\frac{\Delta_{22}}{\Delta_{2}},
$$

where

$$
\begin{aligned}
& \Delta_{2}=\operatorname{det}\left(\begin{array}{cc}
m_{1}+m_{2} & m_{3} \\
n_{2} & n_{1}+n_{3}
\end{array}\right), \quad \Delta_{21}=2 \operatorname{det}\left(\begin{array}{cc}
-p_{1} & m_{3} \\
-p_{2} & n_{1}+n_{3}
\end{array}\right), \\
& \Delta_{22}=2 \operatorname{det}\left(\begin{array}{cc}
m_{1}+m_{2} & -p_{1} \\
n_{2} & -p_{2}
\end{array}\right) .
\end{aligned}
$$


From Eqs. (3.21), (3.23), (3.28), (3.29), we can determine $g_{21}$ and derive the following values:

$$
\begin{aligned}
& c_{1}(0)=\frac{i}{2 \omega^{*} \tau_{1_{0}}}\left(g_{20} g_{11}-2\left|g_{11}\right|^{2}-\frac{\left|g_{02}\right|^{2}}{3}\right)+\frac{g_{21}}{2}, \\
& \mu_{2}=-\frac{\operatorname{Re}\left\{c_{1}(0)\right\}}{\operatorname{Re}\left\{\lambda^{\prime}\left(\tau_{1_{0}}\right)\right\}} \\
& \beta_{2}=2 \operatorname{Re}\left(c_{1}(0)\right) \\
& T_{2}=-\frac{\operatorname{Im}\left\{c_{1}(0)\right\}+\mu_{2} \operatorname{Im}\left\{\lambda^{\prime}\left(\tau_{1_{0}}\right)\right\}}{\omega^{*} \tau_{1_{0}}} .
\end{aligned}
$$

These formulas describe the periodic solutions of Eq. (3.1) at $\tau=\tau_{1_{0}}$ on the center manifold. From the discussion above, we have the following result.

Theorem 3.1 The direction of Hopf bifurcation is determined by the sign of $\mu_{2}$ : if $\mu_{2}>0$ $\left(\mu_{2}<0\right)$, then the Hopf bifurcation is supercritical (subcritical). The stability of the bifurcating periodic solutions is determined by the sign of $\beta_{2}$ : if $\beta_{2}<0\left(\beta_{2}>0\right)$, the bifurcating periodic solutions are stable (unstable). The period of the bifurcating periodic solutions is determined by the sign of $T_{2}$ : if $T_{2}>0\left(T_{2}<0\right)$, the bifurcating periodic solutions increase (decrease).

\section{Numerical examples}

In this section, we present some numerical solutions by using Matlab 7.0 to verify the analytical predictions obtained in the previous section, using the hybrid control strategy to gain control of the Hopf bifurcation of a delayed Lotka-Volterra predator-prey system (1.2).

For comparison, the same parameters in Xu et al. [13] are adopted: $r_{1}=r_{2}=0.5, a_{11}=0.5$, $a_{12}=1, a_{21}=1, a_{22}=1$.

For $\alpha=1$ and $\beta=0$, system (1.3) becomes the uncontrolled system (1.2). The Hopf bifurcation analysis about this uncontrolled system was presented by Xu et al. [13]. It is known that system (1.2) has a positive equilibrium at $E_{*}\left(x^{0}, y^{0}\right)=\left(\frac{2}{3}, \frac{1}{6}\right)$.

For $\tau_{1}=0$, we obtain $\omega_{0} \approx 0.4509, \tau_{2}^{0} \approx 3.357$. From Lemma 2.2 , we know the positive equilibrium $E_{*}\left(x^{0}, y^{0}\right)=\left(\frac{2}{3}, \frac{1}{6}\right)$ is unstable for $\tau_{2} \geq \tau_{2}^{0}$, when $\tau_{2}=3.7>\tau_{2}^{0}$, which is illustrated in Figure 1.

For $\tau_{2}=3$, we obtain $\tau_{1}^{0} \approx 0.6673$, the positive equilibrium $E_{*}\left(x^{0}, y^{0}\right)=\left(\frac{2}{3}, \frac{1}{6}\right)$ is unstable for $\tau_{1} \geq \tau_{1}^{0}$, when $\tau_{1}=0.8>\tau_{1}^{0}$, which is illustrated in Figure 2 .

Now appropriate $\alpha, \beta$ are chosen to control system (1.2). Let us consider the following system:

$$
\left\{\begin{array}{l}
\dot{x}(t)=0.5 x(t)\left[0.5-0.5 x\left(t-\tau_{1}\right)-y\left(t-\tau_{2}\right)\right]+0.1 x\left(t-\tau_{1}\right), \\
\dot{y}(t)=0.5 y(t)\left[-0.5+x\left(t-\tau_{2}\right)-y\left(t-\tau_{1}\right)\right]+0.1 y\left(t-\tau_{1}\right) .
\end{array}\right.
$$

According to the hybrid control strategy, we can easily make the Hopf bifurcation of the uncontrolled system (1.2) disappear, as illustrated in Figures 3 and 4. It is illustrated that the onset of Hopf bifurcation is delayed when the hybrid controller has been incorporated into the model. 

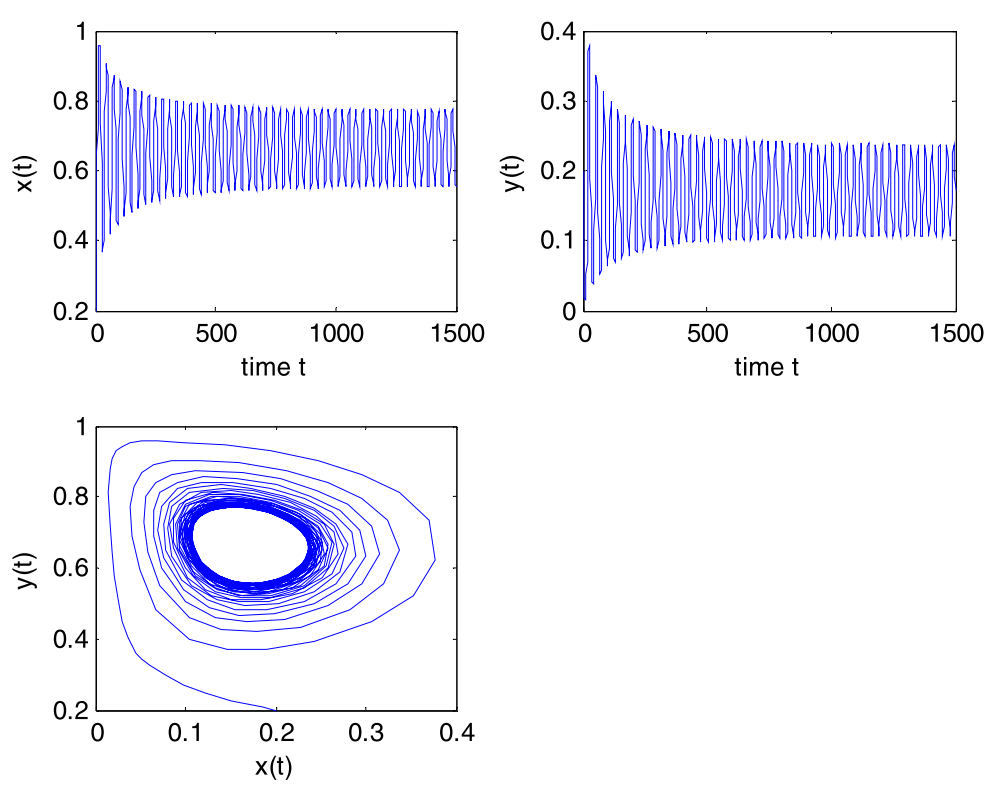

Figure 1 Behavior and phase portrait of an uncontrolled system (1.2) with $\tau_{1}=0, \tau_{2}=3.7>\tau_{2}^{0}$. Hopf bifurcation occurs from the positive equilibrium $E_{*}\left(x^{0}, y^{0}\right)=\left(\frac{2}{3}, \frac{1}{6}\right)$.
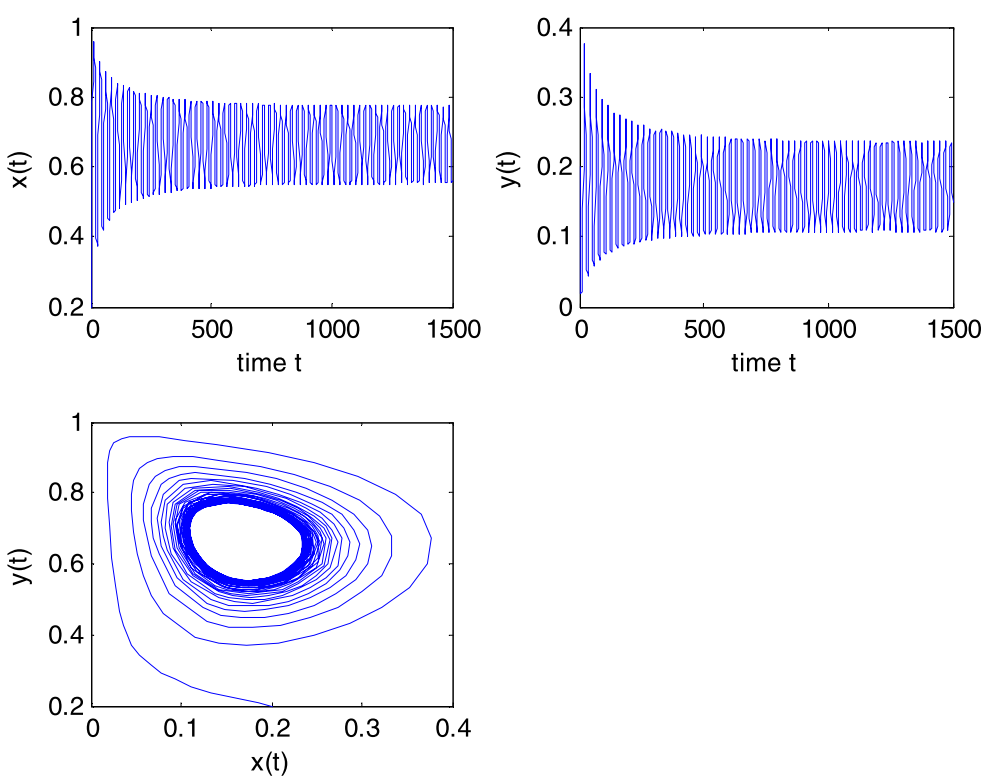

Figure 2 Behavior and phase portrait of an uncontrolled system (1.2) with $\tau_{1}=0.8>\tau_{1}^{0}, \tau_{2}=3.0$. Hopf bifurcation occurs from the positive equilibrium $E_{*}\left(x^{0}, y^{0}\right)=\left(\frac{2}{3}, \frac{1}{6}\right)$.

For $\tau_{1}=0$, we obtain $\omega_{0} \approx 0.1764, \tau_{2_{0}} \approx 4.5037$. From Lemma 2.2, we know the controlled system (1.3) undergoes a Hopf bifurcation at $E^{*}\left(x^{*}, y^{*}\right)$ when $\tau_{2}=\tau_{2_{0}}$. For $\tau_{2}=4.6>$ $\tau_{2_{0}}$, the solution is illustrated in Figure 5 .

For $\tau_{2}=3$, we obtain $\tau_{1_{0}} \approx 2.443$. According to Theorem 2.1, the controlled system (1.3) undergoes a Hopf bifurcation at $\tau_{1_{0}} \approx 2.443$. From Eq. (3.30), we obtain $c_{1}(0)=$ $-0.2954-0.4505 i, \mu_{2}=10.4382, \beta_{2}=-0.5908, T_{2}=0.2761$. According to Theorem 3.1, 

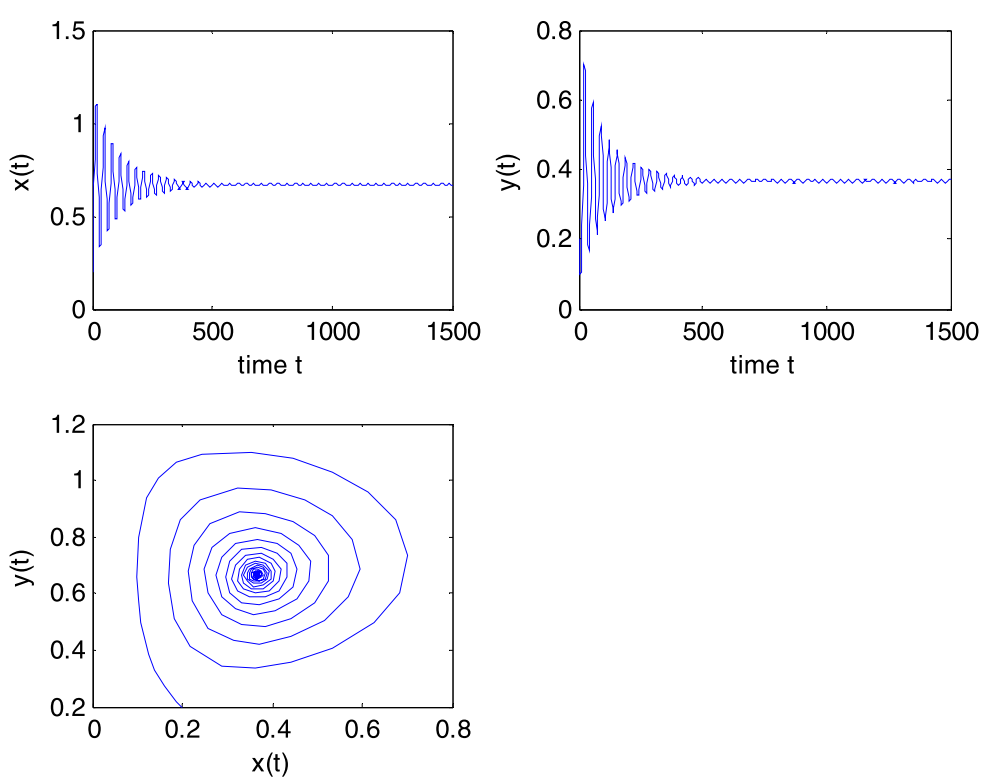

Figure 3 Behavior and phase portrait of a controlled system (1.3) with $\tau_{1}=0, \tau_{2}=3.7>\tau_{2}^{0}$, the Hopf bifurcation disappears.
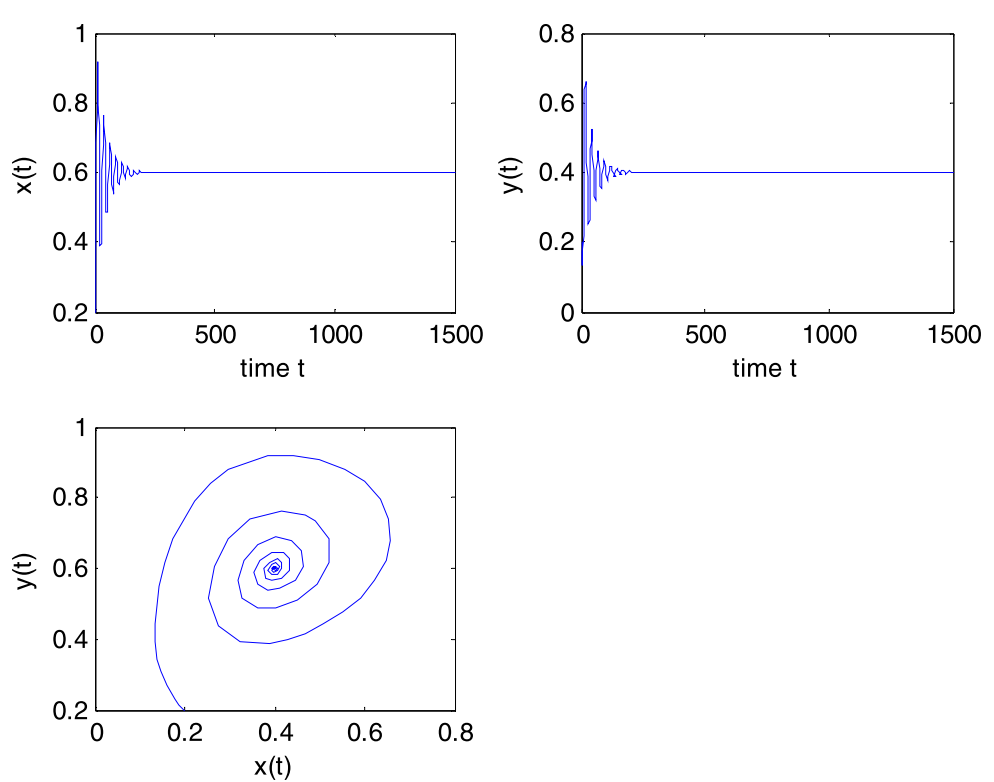

Figure 4 Behavior and phase portrait of a controlled system (1.3) with $\tau_{1}=0.8>\tau_{1}^{0}, \tau_{2}=3.0$, the Hopf bifurcation disappears.

Hopf bifurcation is supercritical, the bifurcation periodic solution exists for $\tau_{1}>\tau_{1_{0}}$ and it is unstable. For $\tau_{1}=2.8>\tau_{1_{0}}$, the solution is illustrated in Figure 6 .

Remark 4.1 For $\alpha=1, \beta=0.1$ in the controlled system (1.3), then we obtain a control model only based on delayed feedback. Take case (b) for example, when $\tau_{1}=0$, by calculation, we have $\omega_{0} \approx 0.2963, \tau_{2_{0}} \approx 2.6612$, the onset of an inherent bifurcation about 

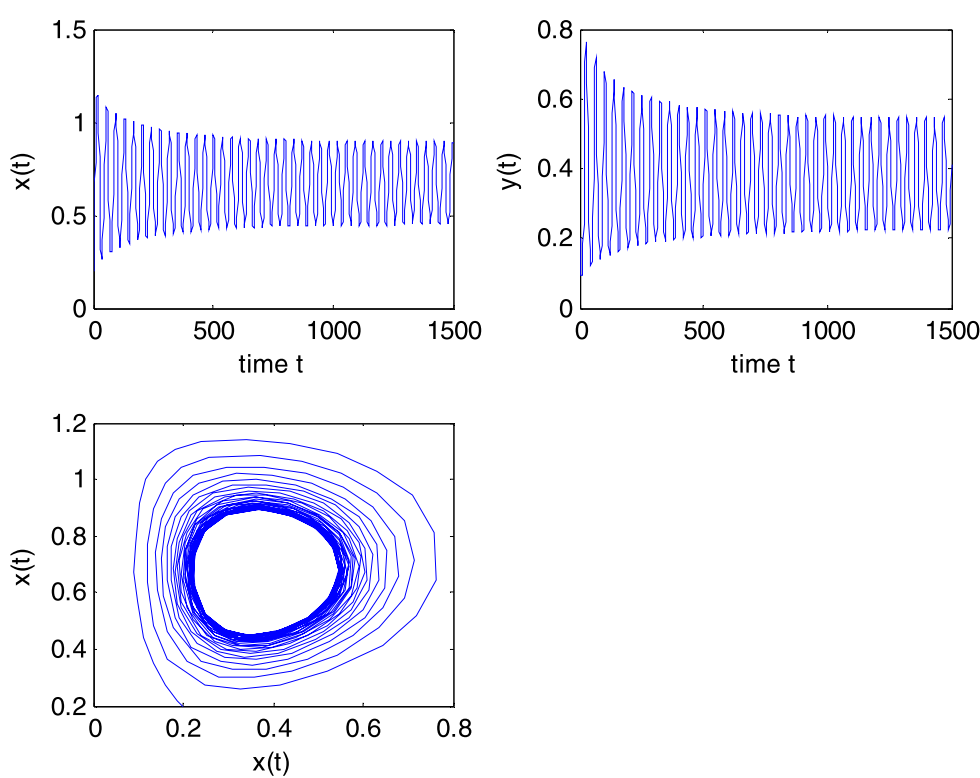

Figure 5 Behavior and phase portrait of a controlled system (1.3) with $\tau_{1}=0, \tau_{2}=4.6>\tau_{2_{0}}$. Hopf bifurcation occurs from the positive equilibrium $E^{*}\left(x^{*}, y^{*}\right)=\left(\frac{2}{3}, \frac{11}{30}\right)$.
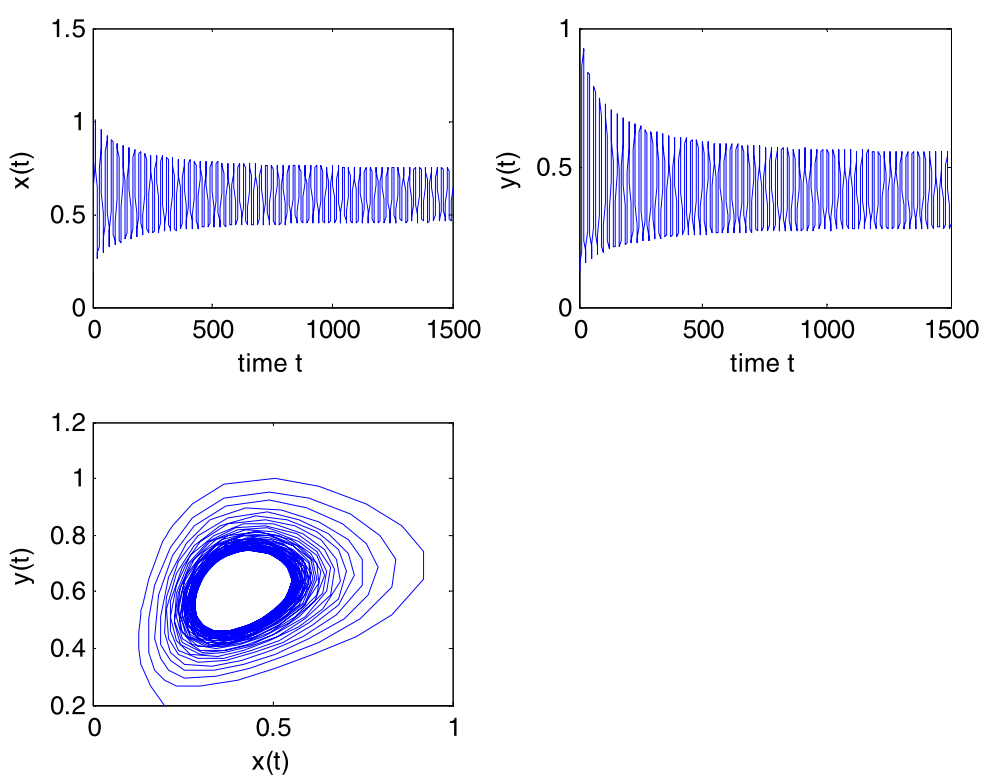

Figure 6 Behavior and phase portrait of a controlled system (1.3) with $\tau_{1}=2.8>\tau_{1_{0}}, \tau_{2}=3.0$. Hopf bifurcation occurs from the positive equilibrium $E^{*}\left(x^{*}, y^{*}\right)=\left(\frac{2}{3}, \frac{11}{30}\right)$.

uncontrolled system (1.2) is not delayed (see Figure 7). It is demonstrated that the hybrid control strategy is more suitable than feedback control of system (1.2) in this paper.

These numerical simulation solutions constitute excellent validation of the new theoretical formulation and analysis presented in this paper. Compared with a general statefeedback control, the hybrid control established here can be more effective in varying the 

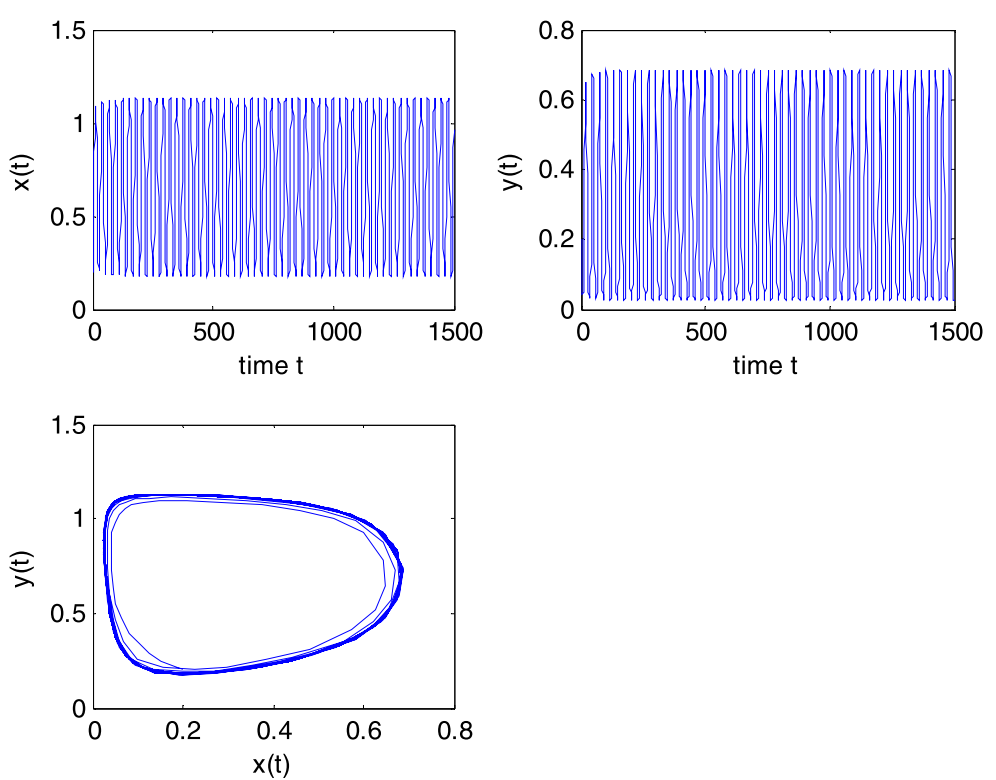

Figure 7 Behavior and phase portrait of a controlled system (1.3) with $\alpha=1, \beta=0.1, \tau_{1}=0$, $\boldsymbol{\tau}_{\mathbf{2}}=\mathbf{3 . 7}>\boldsymbol{\tau}_{\mathbf{2}}^{\mathbf{0}}$. The positive equilibrium $\left(\frac{2}{3}, \frac{4}{15}\right)$ undergoes a Hopf bifurcation.

location of the Hopf bifurcation point while considering parametric disturbance. Therefore, a more practical method for controlling Hopf bifurcation is established.

\section{Conclusions}

In this paper, an efficient hybrid control strategy of Hopf bifurcation for a Lotka-Volterra predator-prey model with two delays has been investigated. By determining an appropriate control parameter, we are able to delay the onset of Hopf bifurcation. For $\tau_{2}=3$, the critical value of delay increases from $\tau_{1_{0}} \approx 0.6673$ to 2.443 . By using the normal form theory and center manifold theorem, the explicit formulas which determine the direction of Hopf bifurcation and stability of the bifurcating periodic solution of the controlled system are derived. The numerical solutions for supercritical Hopf bifurcation and stable bifurcation periodic solutions are in excellent agreement with theoretical analysis. From biology viewpoint, stable bifurcation periodic solutions imply coexistence of both species in an oscillatory mode.

\section{Acknowledgements}

The authors would like to thank the referees for the careful reading of the manuscript and valuable suggestions. This work was supported by the National Nature Science foundation of China (11472116, 11472115), Postgraduate Research \& Practice Innovation Program of Jiangsu Province $(5561190004,4061190009)$ and Qinglan project of Jiangsu.

\section{Competing interests}

The authors declare that they have no competing interests.

\section{Authors' contributions}

All authors contributed equally to the writing of this paper. The authors read and approved the final manuscript.

\section{Publisher's Note}

Springer Nature remains neutral with regard to jurisdictional claims in published maps and institutional affiliations. 


\section{References}

1. Han, W, Liu, MX: Stability and bifurcation analysis for a discrete-time model of Lotka-Volterra type with delay. Appl. Math. Comput. 217, 5449-5457 (2011)

2. He, ZM, Li, B: Complex dynamic behavior of a discrete-time predator-prey system of Holling-III type. Adv. Differ. Equ. 2014(1), Article ID 180 (2014)

3. Khan, AQ: Neimark-Sacker bifurcation of a two-dimensional discrete-time predator-prey model. SpringerPlus 5(1) Article ID 126 (2016)

4. Ren, J, Yu, L, Siegmund, S: Bifurcations and chaos in a discrete predator-prey model with Crowley-Martin functional response. Nonlinear Dyn. 352, 1-23 (2017)

5. Mohammed, EAB, Ahmed, L, Abdelkader, L: Periodic positive solutions of a discrete food chain predator-prey model. MAYFEB J. Math. 2, 21-33 (2017)

6. May, RM: Time delay versus stability in population models with two and three trophic levels. Ecology 54(2), 315-325 (1973)

7. Xu, CJ, Li, PL: Bifurcation behaviors analysis on a predator-prey model with nonlinear diffusion and delay. J. Dyn. Control Syst. 20(1), 105-122 (2013)

8. Banshidhar, S, Swarup, P: Effects of supplying alternative food in a predator-prey model with harvesting. Appl. Math. Comput. 234, 150-166 (2014)

9. Liao, MX, Xu, CJ, Tang, XH: Stability and Hopf bifurcation for a competition and cooperation model of two enterprises with delay. Commun. Nonlinear Sci. Numer. Simul. 19(10), 3845-3856 (2014)

10. Banshidhar, S, Swarup, P: Oscillatory coexistence of species in a food chain model with general Holling interactions Differ. Equ. Dyn. Syst. 22(3), 221-238 (2014)

11. Wei, FY, Fu, QY: Hopf bifurcation and stability for predator-prey systems with Beddington-DeAngelis type functional response and stage structure for prey incorporating refuge. Appl. Math. Model. 40, 126-134 (2016)

12. Joydev, G, Banshidhar, S, Swarup, P: Prey-predator dynamics with prey refuge providing additional food to predator. Chaos Solitons Fractals 96, 110-119 (2017)

13. Xu, CJ, Liao, X, He, XF: Stability and Hopf bifurcation analysis for a Lotka-Volterra predator-prey model with two delays. Int. J. Appl. Math. Comput. Sci. 21(1), 97-107 (2011)

14. Pal, PJ, Mandal, PK, Lahiri, KK: A delayed ratio-dependent predator-prey model of interacting populations with Holling type III functional response. Nonlinear Dyn. 76(1), 201-220 (2014)

15. Wang, XD, Peng, M, Liu, XY: Stability and Hopf bifurcation analysis of a ratio-dependent predator-prey model with two time delays and Holling type III functional response. Appl. Math. Comput. 268, 496-508 (2015)

16. Banshidhar, S, Swarup, P: Effects of additional food in a delayed predator-prey model. Math. Biosci. 261, 62-73 (2015)

17. Song, Y, Xiao, W, Qi, X: Stability and Hopf bifurcation of a predator-prey model with stage structure and time delay for the prey. Nonlinear Dyn. 83, 1409-1418 (2016)

18. Hou, A, Guo, S: Stability and bifurcation in a state-dependent delayed predator-prey system. Int. J. Bifurc. Chaos 26(4), Article ID 1650060 (2016)

19. Cao, J, Yuan, R: Bifurcation analysis in a modified Leslie-Gower model with Holling type II functional response and delay. Nonlinear Dyn. 84(3), 1341-1352 (2016)

20. Dai, Y, Jia, Y, Zhao, H: Global Hopf bifurcation for three-species ratio-dependent predator-prey system with two delays. Adv. Differ. Equ. 2016(1), Article ID 13 (2016)

21. Yang, R, Zhang, C: Dynamics in a diffusive modified Leslie-Gower predator-prey model with time delay and prey harvesting. Nonlinear Dyn. 87(2), 1-16 (2016)

22. $\mathrm{Hu}, \mathrm{D}, \mathrm{CaO}, \mathrm{H}$ : Stability and bifurcation analysis in a predator-prey system with Michaelis-Menten type predator harvesting. Nonlinear Anal., Real World Appl. 33, 58-82 (2017)

23. Yang, Z, Jing, $\mathrm{H}$ : The impact of state feedback control on a predator-prey model with functional response. Discrete Contin. Dyn. Syst., Ser. B 4(3), 607-614 (2004)

24. Li, M, Chen, B, Wang, G, Li, ZH, Liu, W: Hopf bifurcation and nonlinear state feedback control for a modified Lotka-Volterra differential algebraic predator-prey system. In: Fifth International Conference on Intelligent Control and Information Processing, pp. 233-238 (2015)

25. Zhang, F, Li, Y: Stability and Hopf bifurcation of a delayed-diffusive predator-prey model with hyperbolic mortality and nonlinear prey harvesting. Nonlinear Dyn. 88(2), 1-16 (2017)

26. Luo, XS, Chen, GR, Wang, BH: Hybrid control of period-doubling bifurcation and chaos in discrete nonlinear dynamical systems. Chaos Solitons Fractals 18(4), 775-783 (2003)

27. Liu, ZR, Chung, KW: Hybrid control of bifurcation in continuous nonlinear dynamical systems. Int. J. Bifurc. Chaos 15(12), 3895-3903 (2011)

28. Cheng, ZS, Cao, JD: Hybrid control of Hopf bifurcation in complex networks with delays. Neurocomputing 131, 164-170 (2014)

29. Yuan, C, Wu, F: Hybrid control for switched linear systems with average dwell time. IEEE Trans. Autom. Control 60(1), 240-245 (2015)

30. Chen, J, Niu, B, Zhang, J, Chen, X: Hybrid control strategy for the seamless transfer of microgrids. Proc. Chin. Soc. Electr. Eng. 35(17), 4379-4387 (2015)

31. Huang, CD, Cao, JD, Xiao, M: Hybrid control on bifurcation for a delayed fractional gene regulatory network. Chaos Solitons Fractals 87, 19-29 (2016)

32. Ayadathil, J, Venkatesh, J: Hybrid control strategy for matrix converter fed wind energy conversion system. Circuits Syst. 7(10), 3038-3053 (2016)

33. Iyer, VM, Gulur, S, Bhattacharya, S: Hybrid control strategy to extend the ZVS range of a dual active bridge converter In: 2017 IEEE Applied Power Electronics Conference and Exposition (APEC), pp. 2035-2042 (2017)

34. Cai, P, Yuan, ZZ: Hopf bifurcation and chaos control in a new chaotic system via hybrid control strategy. Chin. J. Phys. 55(1), 64-70 (2017)

35. Alfi, A, Kalat, AA, Farrokhnejad, F: Hybrid control strategy applied to chaos synchronization: new control design and stability analysis. Int. J. Dyn. Control 2, 1-8 (2017). doi:10.1007/s40435-017-0343-5 
36. Ruan, S, Wei, J: On the zeros of transcendental functions with applications to stability of delay differential equations with two delays. Dyn. Contin. Discrete Impuls. Syst., Ser. A Math. Anal. 10(6), 863-874 (2003)

37. Hassard, BD, Kazarinoff, ND, Wan, YH: Theory and Application of Hopf Bifurcation. Cambridge University Press, Cambridge (1981)

Submit your manuscript to a SpringerOpen ${ }^{\circ}$ journal and benefit from:

- Convenient online submission

$\checkmark$ Rigorous peer review

Open access: articles freely available online

- High visibility within the field

- Retaining the copyright to your article

Submit your next manuscript at $\gg$ springeropen.com 